\title{
Design and Performance Evaluation of Sensors and Actuators for Advanced Optical Systems (Keynote)
}

\author{
Natalie Clark, NASA Langley Research Center, Hampton Virginia 23681
}

\begin{abstract}
Current state-of-the-art commercial sensors and actuators do not meet many of NASA's next generation spacecraft and instrument needs. Nor do they satisfy the DoD needs for satellite missions, especially micro/nano satellite missions. In an effort to develop advanced optical devices and instruments that meet mission requirements, NASA Langley recently completed construction of a new cleanroom housing equipment capable of fabricating high performance active optic and adaptive optic technologies including deformable mirrors, reconfigurable lenses (both refractive and diffractive), spectrometers, spectro-polarimeters, tunable filters and many other active optic devices. In addition to performance, these advanced optic technologies offer advantages in speed, size, weight, power consumption, and radiation tolerance. The active optic devices described in this paper rely on birefringent liquid crystal materials to alter either the phase or the polarization of the incoming light. Design considerations and performance evaluation results for various NASA applications are presented. Applications presented will include large space telescopes, optical communications, spacecraft windows, coronagraphs, and star trackers.
\end{abstract}

Keywords: Photonics, Adaptive Optics, Tunable Filters, MEMs., MOEMs, Coronagraph, Star Tracker

\section{INTRODUCTION}

Active optic devices are quickly finding their way into advanced optical systems for many aerospace applications. NASA Langley Research Center (LaRC) is at the forefront of development and application of these devices and systems and recently constructed and outfitted a new cleanroom at LaRC dedicated to the fabrication of devices and systems. The active optic elements described in this paper rely on birefringent liquid crystal materials to alter either the phase or the polarization of the incoming wavefront. In this paper we describe the design and performance of several aerospace applications including a star tracker system, coronagraph, optical communications, and large space telescope systems.

\section{LIQUID CRYSTAL DEVICES}

Liquid crystal is a state of matter that is intermediate between a crystalline solid and an amorphous liquid. It may also be viewed as a liquid that has an ordered arrangement of molecules such that a molecular orientation exists. Liquid crystals arise under certain conditions in organic (hydrocarbon) chemicals so that the molecules are sharply anisotropic, that is, they have highly elongated (rod-like) molecules or flattened (disc-like) molecules. A direct consequence of the ordering of the anisotropic molecules is the anisotropy of the mechanical, electric, magnetic, and optical properties.

\subsection{Dielectric Constants.}

Because of the orientation ordering of the rod-like molecules, smectic and nematic liquid crystals are uniaxially symmetric, with the axis of symmetry parallel to the averaged direction of the molecules (direction $\mathbf{n}$, normal to the surface). As a result of the uniaxial symmetry, the dielectric constants differ in value along the preferred axis $\left(\varepsilon_{\|}\right)$and perpendicular to the axis $\left(\varepsilon_{\perp}\right)$. The dielectric anisotropy is defined as

$$
\Delta \varepsilon=\varepsilon_{\|}-\varepsilon_{\perp}
$$

The sign and magnitude of the dielectric anisotropy $\Delta \varepsilon$ are of the utmost importance in the design of devices. All of the devices discussed in this paper utilize the phase modulation property of liquid crystals. Applying an electric field across parallel aligned molecules induces a dipole moment in the molecules. This in turn creates a net toque that tends to align the molecules along the electric field for most rod-like molecules. It is well known in the literature ${ }^{2}$ that the liquid crystal 
molecules are in an orientation distribution where the electrostatic torque exactly balances the elastic restoring torque. A numerical solution is needed to obtain the director distribution. As discussed in the literature ${ }^{2,4}$ for a small distortion, the tilt angle can be expressed mathematically as

$$
\mathrm{k}_{1} \cos ^{2}(\theta)+\mathrm{k}_{3} \sin ^{2}(\theta)(\mathrm{d} \theta / \mathrm{dz})^{2}=\frac{D_{Z}^{2}}{\varepsilon_{\|} \sin ^{2} \theta+\varepsilon_{\perp} \cos ^{2} \theta}-\frac{D_{Z}^{2}}{\varepsilon_{\|} \sin ^{2} \theta_{\max }+\varepsilon_{\perp} \cos ^{2} \theta_{\max }}
$$

where $\theta_{\max }$ is the maximum tilt angle at mid-layer $(\mathrm{z}=\mathrm{d} / 2)$. For example, consider a parallel liquid crystal of ZLI-1646 with a cell gap of $\mathrm{d}=3$ microns. Using the material parameters from Table 1, a numerical integration of Equation (2) yields the maximum tilt angle as a function of applied voltage as shown in Figure 1. The midlayer tilt angle is an increasing function of applied voltage when $\mathrm{V}_{\mathrm{cl}}<\mathrm{V}$. The tilt angle reaches a saturation value of 90 degrees when the applied voltage is much higher than the threshold voltage $\left(\mathrm{V}_{\mathrm{cl}}<<\mathrm{V}\right)^{1-5}$. For a given angle, $\theta_{\max }$, equation (2) can be integrated numerically for the tilt angle distribution $\theta(\mathrm{z})$. Figure $1(\mathrm{a})$ shows the tilt angle distribution $\theta(\mathrm{z})$ for the example ZLI-1646 cell. Referring to the figure, notice that the distortion starts when the applied voltage is greater than the threshold voltage of $1.1961 \mathrm{~V}$. At an applied voltage of $5.3611 \mathrm{~V}$, the midlayer tilt angle is near 90 degrees. Figure 1(b) shows the director tilt angle distribution of a parallel liquid crystal cell of ZLI-1646 with a cell gap of 3 microns, at various applied voltages.

Table 1. Electro-Optical Properties of Liquid Crystal ZLI-1646.

\begin{tabular}{|l|l|}
\hline $\mathrm{T}$ (degree Celsius) & 20 \\
\hline Lambda $(\mathrm{nm})$ & 589 \\
\hline Ordinary Index of refraction & 1.558 \\
\hline Extraordinary Index of Refraction & 1.478 \\
\hline$\varepsilon$ (parallel) & 10.6 \\
\hline$\varepsilon($ perpendicular $)$ & 4.6 \\
\hline $\mathrm{k} 1\left(10^{-12} \mathrm{~N}\right)$ & 7.7 \\
\hline $\mathrm{k} 2\left(10^{-12} \mathrm{~N}\right)$ & 4 \\
\hline $\mathrm{k} 3\left(10^{-12} \mathrm{~N}\right)$ & 12.2 \\
\hline Nematic range (degree Celsius) & $-20-60$ \\
\hline Rotational Viscosity (mPa $\mathrm{s})$ & 160 \\
\hline
\end{tabular}

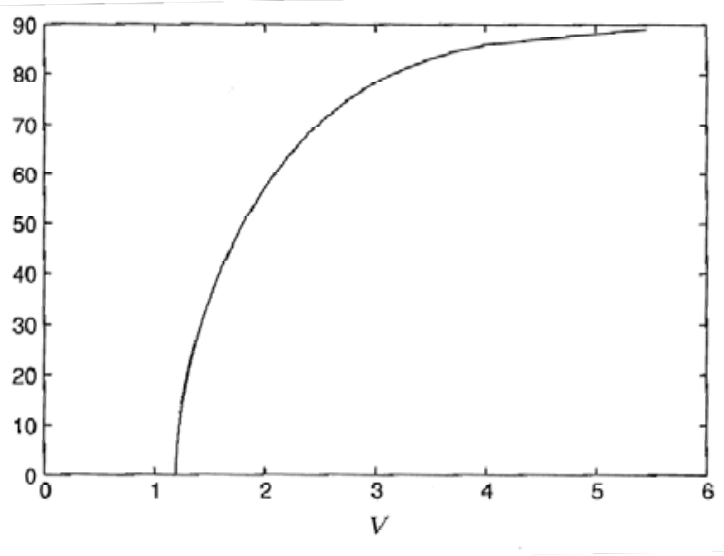

(a)

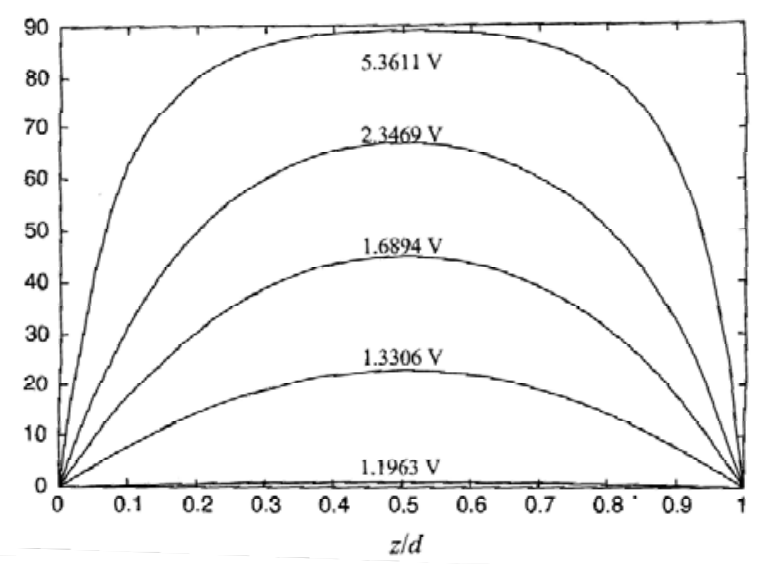

(b)

Figure 1. Operation of a liquid crystal cell of ZLI-1646 with 3 micron cell gap. (a) Director tilt angle distribution of a parallel liquid crystal cell (b) director tilt angle distribution at various applied voltages. 


\section{ARRAY OF LIQUID CRYSTAL CELLS}

NASA LaRC has developed a 1024 x 768 array liquid crystal device with a metalized silicon backplane (commercially obtained) shown below in Figure 2. Such a device is often referred to as a liquid crystal on silicon (LCOS) device. The LCOS panel is basically an electronically controlled phase shifter device with the rubbing direction along the y axis as shown in Figure 2.

Table 2. Liquid Crystal On Silicon (LCOS) Device

\begin{tabular}{|l|l|}
\hline Cell Type & Electronic controlled Phase Shifter \\
\hline Active Area & $20 \mathrm{~mm}$ x $15 \mathrm{~mm}$ \\
\hline Resolution & $1024 \times 768$ \\
\hline Fill Factor & $96.0 \%$ \\
\hline Bit Depth & $8 \mathrm{bit}(256$ gray scale $)$ \\
\hline Temporal Bandwidth & $60 \mathrm{~Hz}$ \\
& \\
\hline Effective Stroke Length & $635 \mathrm{~nm}$ \\
\hline Liquid Crystal Layer Uniformity & $1 / 10$ lambda Peak to Valley at $632.8 \mathrm{~nm}$ \\
\hline Operation Wavelength & $632.8 \mathrm{~nm}$ \\
\hline Beam Steering Range & $\begin{array}{l}+/-4 \text { mrad on both X and Y axis } \\
\text { Steering Efficiency }>80.3 \%\end{array}$ \\
\hline Steering Accuracy & Better than 10 urad \\
\hline
\end{tabular}

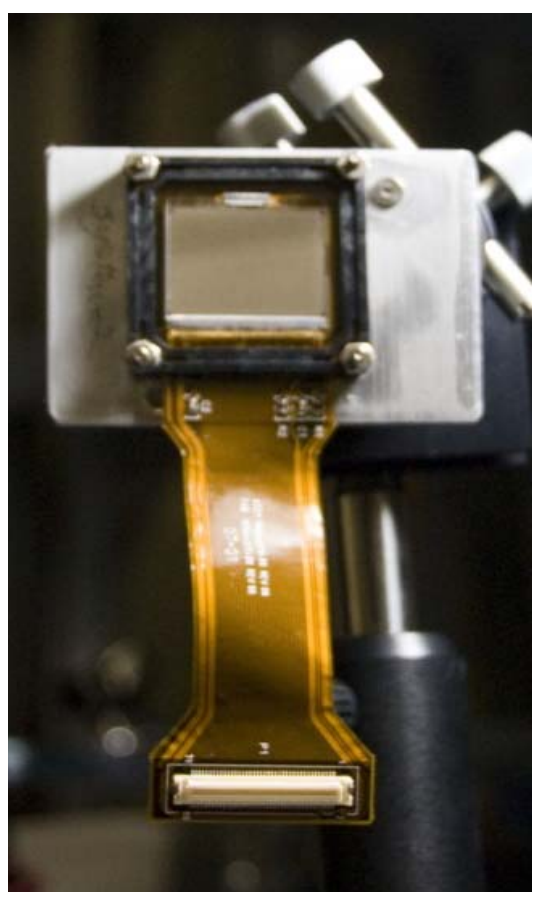

Figure 2. Array of phase shifters. The array of phase shifters consists of a 1024 x 768 array of pixels. Each pixel is a single layer phase shifter. 


\subsection{Phase Shifting Calibration of the LCOS Array}

The LCOS device was calibrated using a Zygo Interferometer. A schematic of the experimental layout is shown in Figure 3. A sequence of phase maps for each pixel at each gray level was acquired. The non-uniformity was less than 1/10 lambda; hence, a simple polynomial interpolation of the phase, $Y$, can be used to represent the pixel controller plant model. Any requirement for increased wavefront accuracy is beyond the capability of commercial interferometers and new more sophisticated wavefront sensors would need to be developed.

$$
\mathrm{Y}=\mathrm{p} 1 * \mathrm{x}^{\wedge} 4+\mathrm{p} 2 * \mathrm{x}^{\wedge} 3+\mathrm{p} 3 * \mathrm{x}^{\wedge} 2+\mathrm{p} 4 * \mathrm{x}+\mathrm{p} 5
$$

For our LCOS device $\mathrm{p}_{1}=-82.521 ; \mathrm{p}_{2}=425.35 ; \mathrm{p}_{3}=-484.04 ; \mathrm{p}_{4}=-49.964$ and $\mathrm{p}_{5}=213$.

\subsection{Near Field Performance Evaluation of the LCOS Array}

Shown in Figure 4 is one frame of the interferogram taken by the Zygo interferometer. Referring to the interferogram, the wavefront distortion between any bright fringes is 1 wavelength at $623.8 \mathrm{~nm}$, or $1 / 2$ wavelength of the surface height difference. The whole device is not a flat surface but has a large spherical shape. The main reason for this is the whole cell is glued to a metal heat sink, which is not flat. When the glue hardens, some tension is formed and thus bends the entire cell. Fortunately, this bending is spherical and simply focusing the optics to compensate for the defocus induced by the device bending results in a very flat wavefront in the optic exit pupil. The liquid crystal device was placed on a micrometer stage capable of moving along the optic axis. The phase shift profiles were recorded by the interferometer. Assuming the irradiance on a given pixel is $\mathrm{I}_{1}(\mathrm{x}, \mathrm{y}), \mathrm{I}_{2}(\mathrm{x}, \mathrm{y}), \mathrm{I}_{3}(\mathrm{x}, \mathrm{y}), \mathrm{I}_{4}(\mathrm{x}, \mathrm{y})$, and $\mathrm{I}_{5}(\mathrm{x}, \mathrm{y})$ for each of the five phase shift stage positions, the wavefront phase profile is given by

$$
\Phi=\tan ^{-1}\left(2\left(\mathrm{I}_{4}-\mathrm{I}_{2}\right) /\left(2 * \mathrm{I}_{3}-\mathrm{I}_{1}-\mathrm{I}_{5}\right)\right) / 2
$$

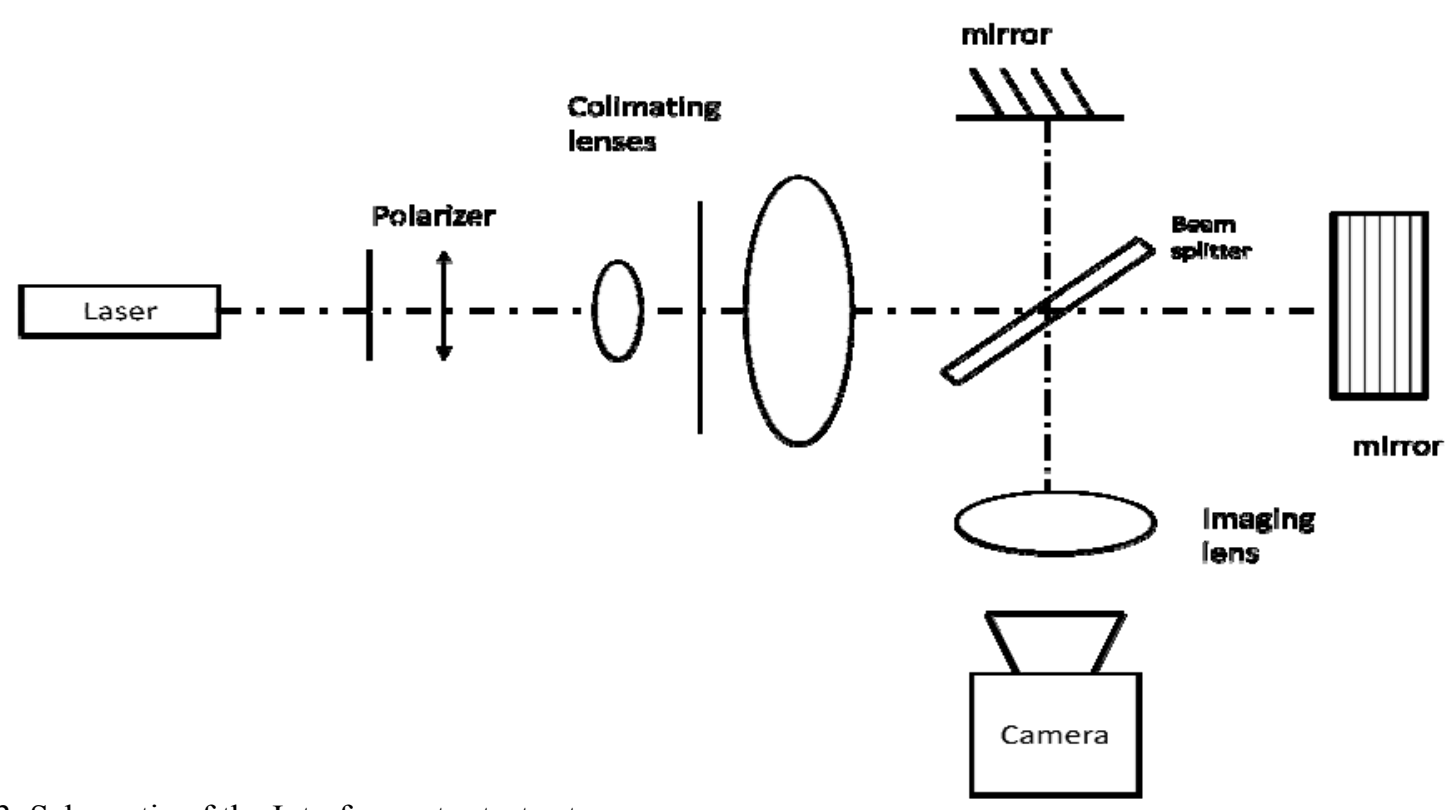

Figure 3. Schematic of the Interferometer test set up. 


\subsection{Far Field Characterization}

The far field beam quality was also measured to characterize the LCOS device. A small telescope with effective focal length of $1170 \mathrm{~mm}$ and a diameter of $76.2 \mathrm{~mm}$ was used. The $650 \mathrm{~nm}$ wavelength laser beam was reflected off the LCOS device and focused. The irradiance profile was measured with a CCD camera. Figure 5 shows the beam profile of the focused laser beam. In order to evaluate the LCOS compensation for the far field, the beam was expanded to form a $1 / \mathrm{e}^{2}$ waist of $1.02 \mathrm{~mm}$ to $1.02 \times 150 / 16.5=9.27 \mathrm{~mm}$. The beam was then focused and measured to have a beam waist of $\mathrm{w}=$ 107.3 microns. The Strehl ratio is a useful metric to characterize the compensation performance. Figure 6 shows an uncompensated and compensated beam. The Strehl ratio is defined as the normalized peak intensity on diffraction focus. The Strehl ratio of the compensated beam was about 100 times higher than the non-compensated beam. Ideally a diffraction limited compensation would yield a Strehl ratio larger than 0.84. One of the main reasons the LCOS is not quite diffraction limited was because the aluminum mirror on the panel did not have a dielectric coating which resulted in a low reflectivity of $80.4 \%$. Note that the Strehl ratio is normalized to the total energy to measure the percent energy contained in the Airy pattern ${ }^{6}$. The beam waist after beam expansion was $9.27 \mathrm{~mm}$, compared with the $20 \times 15 \mathrm{~mm}$ aperture of the LCOS device. The long axis of the LCOS device is always defined as the Y axis and the short axis is defined as the $\mathrm{X}$ axis. The beam waist after beam expansion was $9.27 \mathrm{~mm}$, which means the entire beam is contained within the aperture of the LCOS device of $20 \times 15 \mathrm{~mm}$. The long axis of the LCOS device is always defined as the Y axis and the short axis is defined as the $\mathrm{X}$ axis.

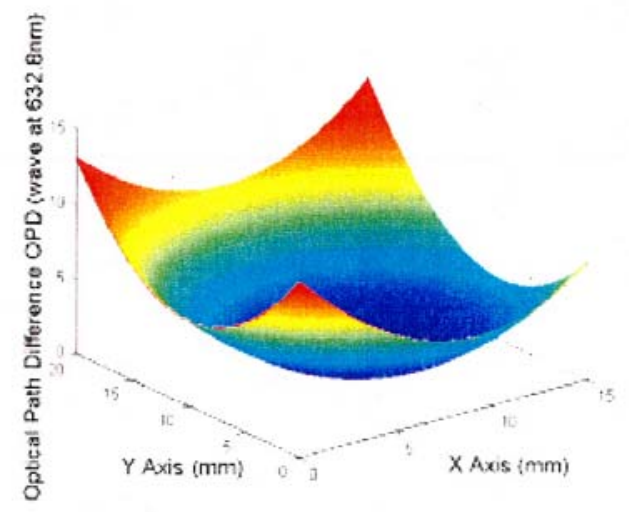

(a)

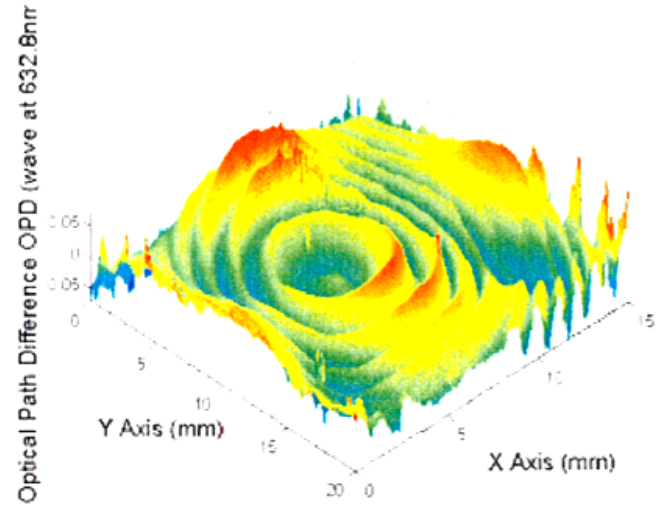

(b)

Figure 4. Interferogram of the LCOS device before and after compensation of defocus aberration. (a) is the wavefront of a defocus aberration introduced by moving the collimating lens to induce a small wavefront aberration of defocus. (b) Surface map of the wavefront residual aberrations after compensation.

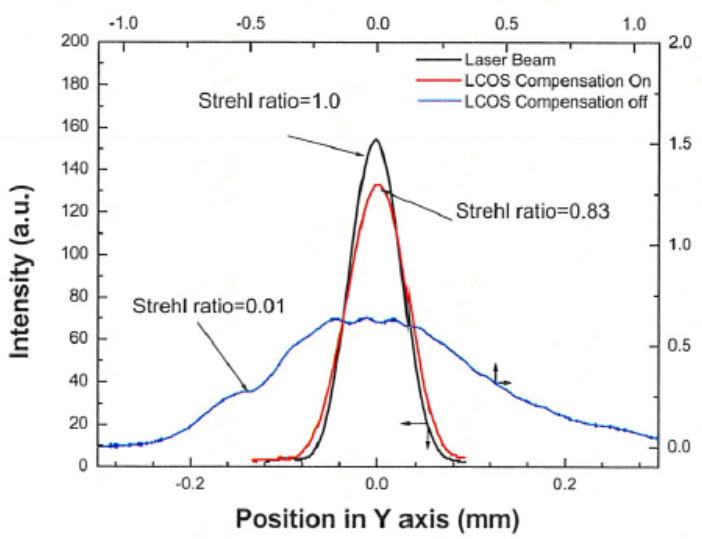

Figure 5. Beam profile of a focused laser beam. The laser beam is shown in black, the LCOS with compensation on is shown in red and the uncompensated laser beam is shown in blue. 

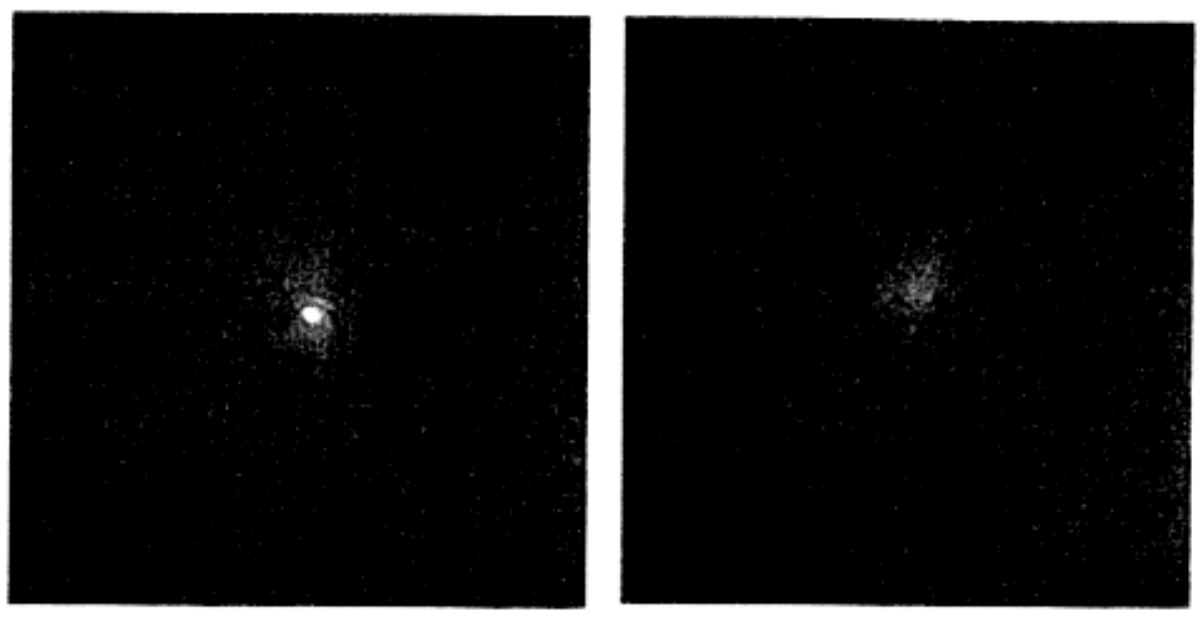

Figure 6. Compensated Beam using LCOS device. (a) compensated beam using the LCOS device as a phase modulator (b) uncompensated beam.

\section{ACHROMAT PHASE SHIFTER}

The phase retardation of a single plate of birefringent crystal material is given by

$$
\Gamma=2 \pi\left(\mathrm{n}_{\mathrm{s}}-\mathrm{n}_{\mathrm{f}}\right) \mathrm{d} / \lambda
$$

where $d$ is the thickness of the plate and $n_{s}$ and $n_{f}$ are the slow and fast indices, respectively and $\lambda$ is the wavelength of light. For a uniaxial plate, $\left(\mathrm{n}_{\mathrm{s}}-\mathrm{n}_{\mathrm{f}}\right)=\left(\mathrm{n}_{\mathrm{e}}-\mathrm{n}_{\mathrm{o}}\right)$. According to Equation (5) the phase retardation is strongly dependent on the wavelength via the $(1 / \lambda)$ factor. Figure 7 shows the phase retardation of an achromatic phase shifter designed and fabricated at Langley. The design is based on the Pancharatnam analysis ${ }^{2}$. Pancharatnam introduced a convention for comparing the phases of two beams of light in different states of polarization. Based on his suggestion, a relative phase between two different polarization states $\mathbf{E}_{\mathrm{A}}$ and $\mathbf{E}_{\mathrm{B}}$ can be expressed as the irradiance, I, of the two interfering polarized beams expressed as vectors $\mathbf{E}_{\mathrm{A}}$ and $\mathbf{E}_{\mathrm{B}}$

$$
I=\left(\boldsymbol{E}_{A}+\exp (i \chi) \boldsymbol{E}_{B}\right)^{*} \cdot\left(\boldsymbol{E}_{A}+\exp (i \chi) \boldsymbol{E}_{B}\right)
$$

Where * denotes the complex conjugate and $\cdot$ denotes the dot product of vector $\mathbf{E}_{\mathrm{A}}$ and $\mathbf{E}_{\mathrm{B}}$

Figure 7 shows a schematic of the achromat phase shifter design that can be implemented in many NASA and DoD applications. These applications include star sensors, coronagraphs, spectro-polarimeters, and optical communication systems as described in Section 5 of this paper. Referring to Figure 7, the achromat phase shifter consists of a half wave plate sandwiched between two dynamically controlled phase shifters. The orientation angles of the dynamic phase shifters and half wave plate are chosen to minimize the phase variation as a function of wavelength. For example, if a 90 degree phase shift is desired at $550 \mathrm{~nm}$ the dynamic cell is operated at ${ }_{2}=45$ degrees. As shown in Figure 8 the phase shift is 90 degrees at $550 \mathrm{~nm}$ as designed for both the single cell and achromat designs. The dynamic cells directors are oriented at approximately 67.5 degrees with respect to the half waveplate. Figure 8 (a) shows the performance of a single cell phase shifter operating at 90 degrees at 550nm design. Referring to Figure 8(a) the phase shift for a single cell phase shifter varies significantly with wavelength. For a wavelength of $450 \mathrm{~nm}$ the phase shift is around 110 degrees which is 
20 degrees higher than desired ( 90 degrees is desired for all wavelengths). At a wavelength of $650 \mathrm{~nm}$ the phase shift of 78 degrees is 12 degrees smaller than desired. The achromatic design shown in Figure 8(b) shows the significantly improved performance. Referring to figure $8(\mathrm{~b})$, the phase shift at $450 \mathrm{~nm}$ wavelength is approximately 86 degrees (only 4 degrees less than desired). At a wavelength of $650 \mathrm{~nm}$ the phase shift is 88 degree (only 2 degrees less than desired). The phase shifter is quite insensitive to variations in wavelength when compared to the single cell design. Additional layers can be added to further enhance the design.

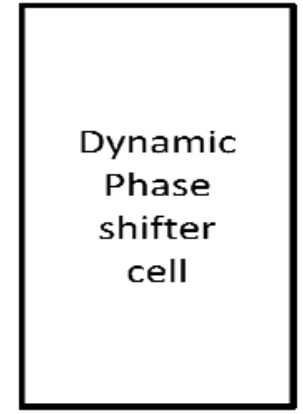

1

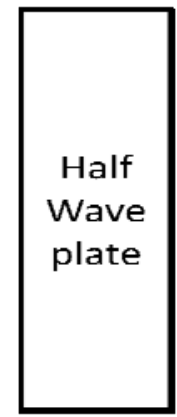

2

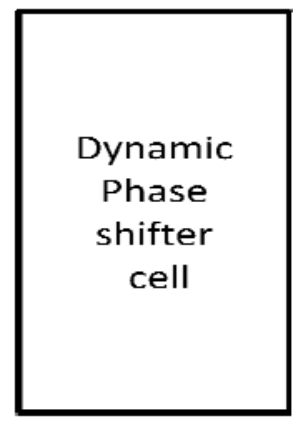

1

Figure 7. Achromatic phase shifter (a) design of an achromatic phase shifter. The orientation angle of each cell, $\quad 1 \quad$ and 2 , is chosen to yield the best achromatic (smallest phase shift variation as a function of wavelength).
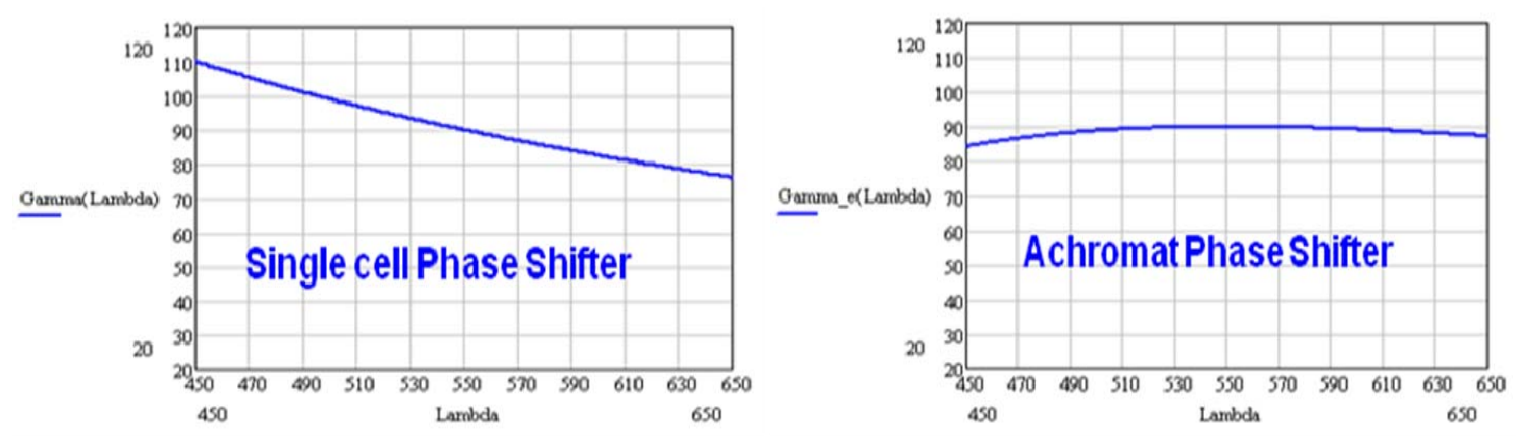

$\mathrm{n}$

Figure 8. Performance of phase shifter (a) Single cell dynamic phase shifter operating at 90 degrees phase shift at 550 $\mathrm{nm}$ (b) Achromat phase shifter

\section{APPLICATIONS OF ACROMAT PHASE SHIFTERS}

In this section some advanced optical applications using a novel phase shifter device are described along with a summary of the requirements needed for NASA missions. Design considerations and performance evaluation results for various NASA applications are presented. Applications presented include large space telescopes, optical communications, spacecraft windows, coronagraphs, and star trackers. We anticipate reporting on each of these systems in future publications. 


\subsection{Intelligent Star Tracker System \& Optical Communications}

Prior publications have described our research efforts to develop a light-weight star sensor for autonomous Guidance Navigation and Control GNC and rendezvous and docking applications. ${ }^{79}$ Figure 9 (a) shows a schematic of the current intelligent star sensor along with a prototype developed that weighs less than 1.84 pounds. Referring to Figure 9, the star sensor is a folded catadioptic telescope with a thin corrector plate to correct for aberration and enable a $24 \times 24$ degree field of view. Currently in development is an array of hexagon shaped phase plates that will allow real-time phase shifting as shown in Figure 10. The current intelligent star tracker design has an accuracy exceeding 5 arc seconds. The array of achromat phase shifters will enable the performance to exceed 1 arc second.

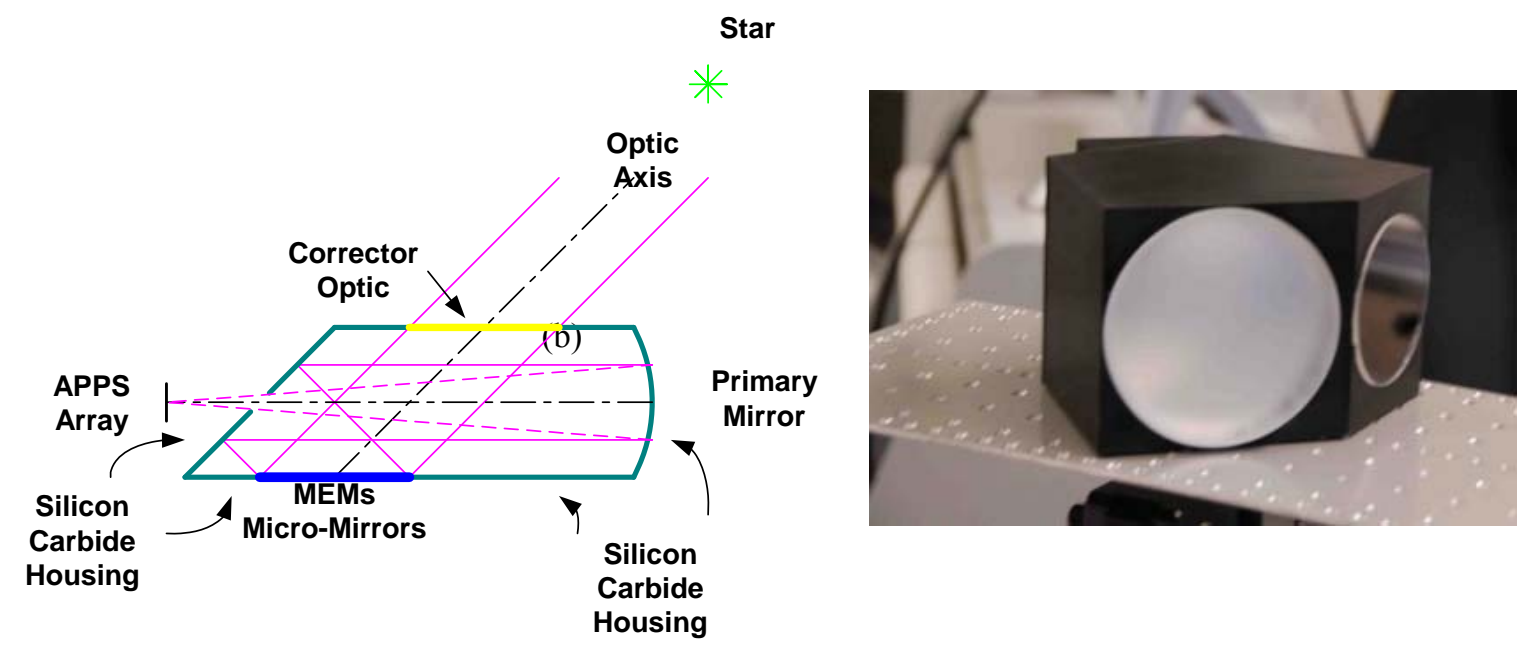

Figure 9. Intelligent Star sensor (a) schematic of the optical system (b) prototype intelligent star sensor

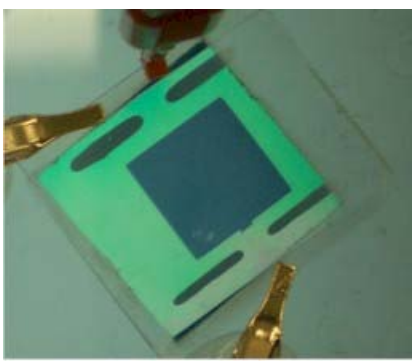

(a)

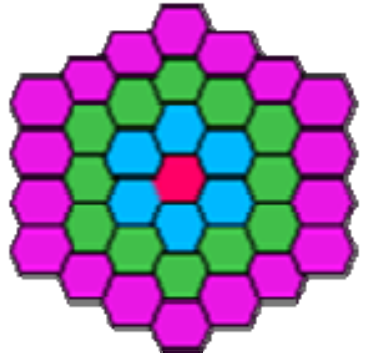

(b)

Figure 10 Liquid crystal phase modulator (a) single phase modulator cell fabricated at NASA LaRC and (b) Corrector plate architecture of hexagonal single cell phase modulator 


\subsection{Spectro-Polarimeter and Plastic Windows}

We are currently investigating the use of plastic windows to reduce the weight of crewed spacecraft. ${ }^{10}$ Plastic windows are birefringent as shown in Figure 11. Since they are thick, 0.25 inch or more, the retardance of some of them can be quite large, on the order of $500 \mathrm{~nm}$ or more. Hence, we are developing a spectro-polarimeter based on the Pancharatnam techniques discussed previously in Section 4. Figure 11(a) shows a schematic of the spectro-polarimeter that uses an achromat phase shifter. Figure 11 (c) shows the retardance vs. wavelength of a plastic window with very low retardance. This shows that our system can not only measure large amounts of birefringence but also has a large dynamic range capable of measuring the retardance with an accuracy near the noise limit of the detector, approximately $1 \mathrm{~nm}$ retardance. We will be presenting more detailed design and performance evaluation data in a future publication.

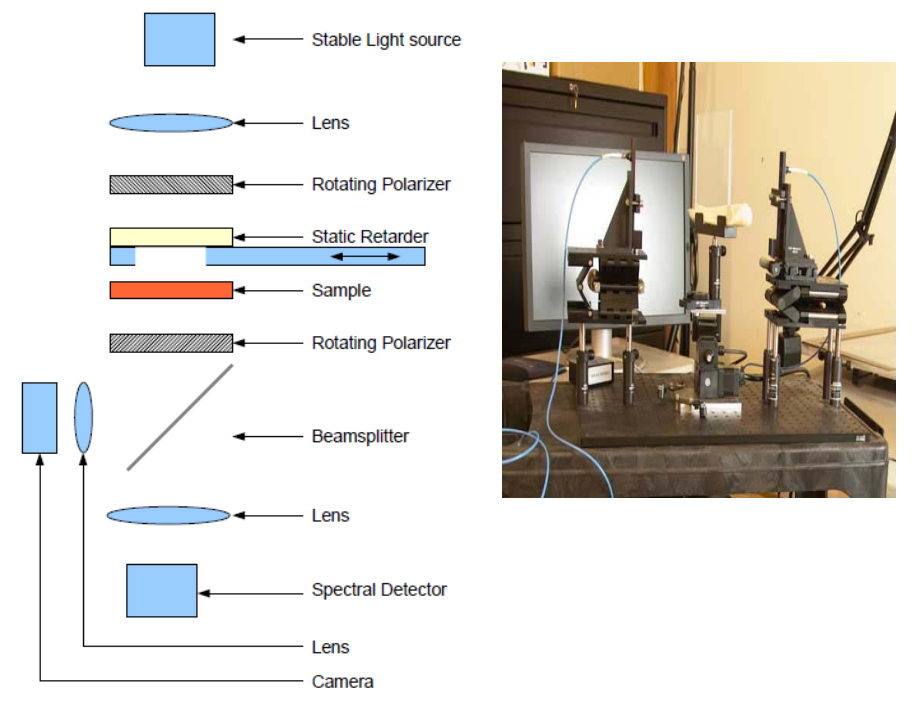

(a) (b)
Spectral Retardance vs. Wavelength (nm)

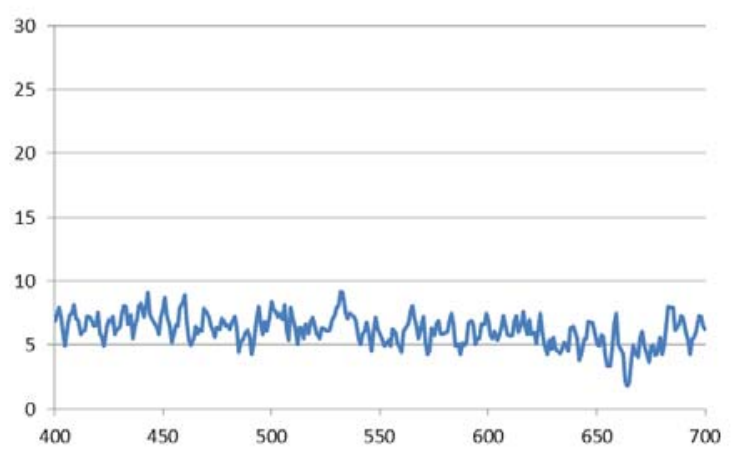

Figure 11. Spectral retardance of plastic windows. (a) schematic of the spectro-polarimeter (b) the window placed in the spectro-polarimeter (c) spectral birefringence measurement of window with small birefringence showing the good sensitivity of our instrument is near the detector noise limit.

\subsection{Coronagraph}

A coronagraph is a telescopic attachment designed to block out the direct light from a star so that nearby objects, which otherwise would be hidden in the star's bright glare, can be resolved. The detection and characterization of planets that orbit stars other than our Sun (referred to as "exoplanets" or "extrasolar planets") will profoundly improve our understanding of the evolution of the universe. ${ }^{11-12}$ Direct detection of exoplanets, however, poses a difficult problem because of the huge contrast between the planet and the star it orbits. The contrast between an exoplanet and its parent star is often quoted a 1 part in $10^{8}$ for Jupiter and 1 part in $10^{10}$ for Earth ${ }^{11-12}$. NASA, as are many in the coronagraph community, is targeting a contrast requirement of $10^{12}$. Not only must such a contrast be achieved, but it must also be maintained over a broad wavelength range. NASA is currently investigating the use of novel achromat phase shifting technologies for use in coronagraph systems. Referring to Figure 12, there are several components that are based on achromat phase shifter technology. The phase plates and shearing mechanism shown in Figure 12 (a) both can benefit from achromat performance as the coronagraph is broadband. The achromat phase shifters shown in the optical breadboard shown in Figure 12 (b) is another place the coronagraphs can benefit from the technology presented in this paper. Traditional designs are achromatic over a very short bandwidth of 10 or $20 \mathrm{~nm}^{11-12}$. The technique shown in this paper can achieve a bandwidth of $200 \mathrm{~nm}$ over a performance range of 400-600 $\mathrm{nm}$. 
Another very serious concern common to all coronagraph systems is the issue with the polarization effects associated with the metal coatings of the telescope mirror. ${ }^{11}$ For large telescopes, such as those planned for NASA Exoplanet missions, light hits the telescope mirror at large angles of 20 to 30 degrees. At such large angles there is a serious polarization and phase concern associated with light reflecting from metal thin films. The metal film has a complex index of refraction that varies with the angle and wavelength of light. This causes the beam of light to change in polarization, amplitude and phase. Upon reflection the polarization components of the beam of light are rotated. The amount of rotation depends on the angle of incidence and wavelength. To further complicate matters, the metal thin film coatings have regions of anisotropies inherent in the metal deposition process. ${ }^{11-12}$ The anisotropies cause additional random like variations in birefringence which are also dependant on the angle light hits them and are strongly wavelength dependent. These issues are serious and are well known problems in the coronagraph community ${ }^{11-12}$. In addition to the primary mirror, deformable mirrors also have thin-film metallic layers inducing local anisotropy that introduces unwanted polarization effects ${ }^{11}$. These polarization and phase effects have typically been overlooked because of their perceived complexity. This is coupled with the lack of fabrication facilities capable of manufacturing precise optical phase and polarization correctors. NASA is developing a device for broad band compensation of the polarization and anisotropy in high contrast coronagraph systems using novel broadband synthesized polarization/phase correctors based on achromat phase shifter technology. Each polarization component will need an acromat phase shifter. We are able to develop a pixilated device where each pixel consists of a phase shifter for each polarization state.
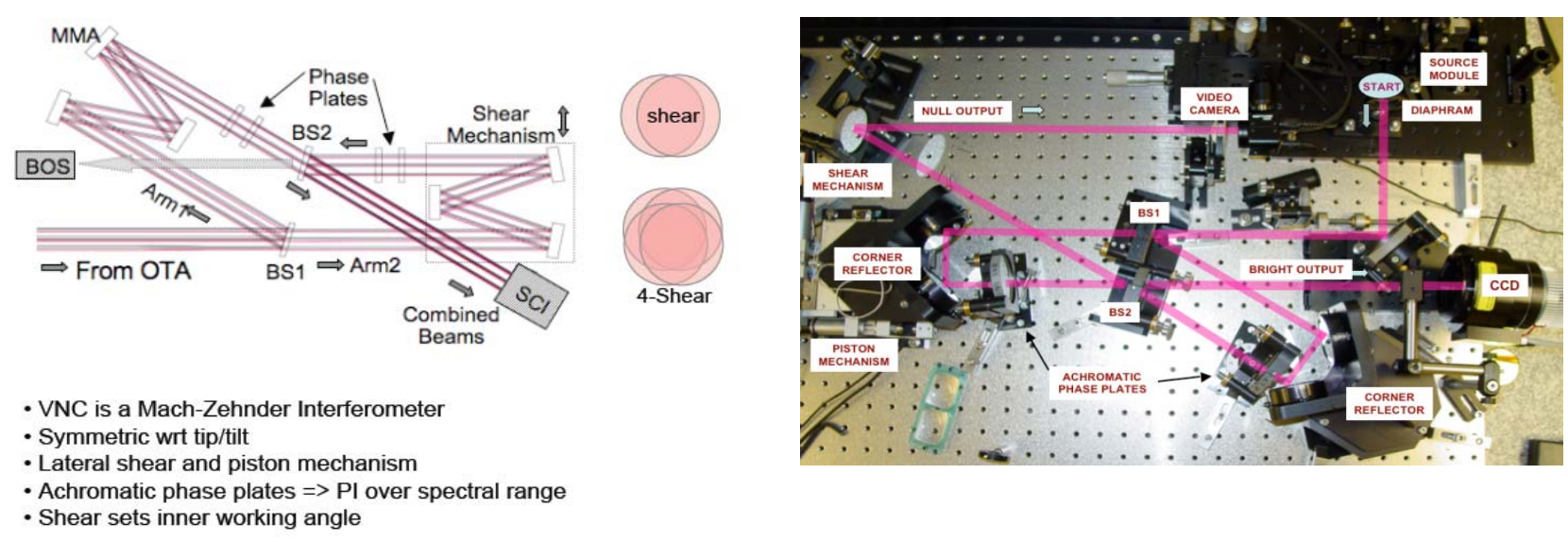

Figure 12. NASA EPIC Coronagraph system. (a) optical schematic and (b) optical breadboard of the visible nulling coronagraph system.

\section{ACKNOWLEDGEMENTS}

Thanks to Phil Bos for helping us obtain some silicon backplanes for the LCOS device as well as providing his expertise in liquid crystal device modeling and testing. Thanks to Rick Lyons and Jim Breckinridge for their expertise regarding coronagraph system design and requirements for achromat polarization corrector plates. 


\section{REFERENCES}

[1] S.T Wu, Nematic Liquid Crystals. Spatial Light Modulator Technology- Materials, Devices and Applications, ed U. Efron, Vol 1. Marcel Dekker, Inc., New York, 1995.

[2] S. Pancharatnam Proc Indian Acad. Sci A 44, 247 (1956).

[3] Pochi Yeh and M Paukshto, "Molecular crystalline thin film E-polarizer," Mol. Materials, Vol. 14, pp 1-19 (2001).

[4] J. Chen, K. -H. Kim, J. -J. Jyu, J. H. Souk, J. R. Kelly and P. J. Bos, Proc. SID, paper 21.2 (1998).

[4] D. W. Berreman "Optics in Stratified and Anisotropic Media: 4 x 4-Matrix Formulation" J. Opt. Soc. Am., 62, 502510 (1972).

[5] S. Chandrasekhar "Liquid Crystals", Cambridge University Press: 1994.

[6] Robert K Tyson "Principles of Adpative Optics”, Academic Press; 2 edition (October 13, 1997).

[7] Natalie Clark an Paul Furth 'Intelligent Star Tracker” SPIE 1999.

[8] Crandall Charles, Clark, Natalie, and Davis, Patricia "Tunable optical filters for space exploration", SPIE Nanophotonics and Macrophotonics for space environments Vol. 6713 Sep 2007.

[9] Clark, Natalie "Advanced Optical Technologies for space exploration (invited) SPIE Nanophotonics and Macrophotonics for space environments Vol. 6713 Sep 2007.

[10] NASA CXP72407, “Optical Requirements Document for Spacecraft windows”, pending approval, January 2011.

[11] Breckinridge, James and Oppenheimer, Ben "Polarization Effects In Reflecting Coronagraphs for White-Light Applications in Astronomy”, The Astrophysical Journal, 600:1091-1098, January 2004

[12] Richard G. Lyon, Mark Clampin, Peter Petrone, Patrick Thompson, Timothy Madison, Robert Woodruff, Charley Noecker, Steve Kendrick Technology Advancement of the Visible Nulling Coronagraph, International Conference in the Spirit of Lyot, Paris France, October 2010 